\title{
La cultura gerencial con respecto a la conducción efectiva de las organizaciones del deporte profesional: El caso Venezuela
}

\section{Management culture with respect to the effective management of professional sports organizations: The Venezuela case}

Summar Alfredo Gómez Barrios

Universidad Iberoamericana del Ecuador UNIB.E, Ecuador

Autor para correspondencia: summar.gomez@gmail.com

Fecha de recepción: 29 de Agosto 2017 - Fecha de aceptación: 15 de Enero de 2018

Resumen: El interés de este artículo es conocer la visión que tienen los dirigentes del deporte de la cultura gerencial con respecto a la conducción efectiva de las organizaciones del deporte: El caso Venezuela, haciendo énfasis en las cinco ligas profesionales que operan actualmente, como son las ligas de baloncesto, béisbol, futbol, polo acuático y voleibol. Para tales propósitos, fueron entrevistados doce actores sociales que ejercen funciones gerenciales en estas ligas, con el necesario potencial y relevancia en cuanto a su estatus, atributos, capacidades y conocimientos para ser consultados. La metodología estuvo enmarcada dentro del paradigma cualitativo, apoyada en un nivel de investigación descriptivo y un diseño de investigación de campo. Para la captura, procesamiento y análisis de los datos que surgieron de las entrevistas en profundidad, el estudio estuvo soportado en la teoría fundamentada. Los resultados de este estudio permitieron constatar que los gerentes pertenecientes a las organizaciones del deporte profesional en Venezuela, esperan una concepción filosófica que esté determinada por un conjunto de aspectos de naturaleza intangible como las actitudes, los valores, las emociones, las rutinas, entre otros, a los efectos de facilitar la interacción dentro y entre los grupos existentes en estas organizaciones, aspectos que deben estar adecuadamente alineados con la misión, visión y valores organizacionales.

Palabras Clave: gerencia; cultura gerencial; organizaciones; deporte profesional

\begin{abstract}
The interest of this article is to know the vision that have the leaders of the sport of the managerial culture with respect to the effective conduction of the organizations of the sport: The case Venezuela, emphasizing in the five professional leagues that operate at the moment, as the leagues of Basketball, baseball, soccer, water polo and volleyball. For these purposes, twelve social actors were interviewed who exercise managerial functions in these leagues, with the necessary potential and relevance in terms of their status, attributes, skills and knowledge to be consulted. The methodology was framed within the qualitative paradigm, supported by a level of descriptive research and a field research design. For the capture, processing, and analysis of the data that emerged from in-depth interviews, the study was supported by grounded theory. The results of this study showed that managers belonging to professional sports organizations in Venezuela expect a philosophical conception that is determined by a set of intangible aspects such as attitudes, values, emotions, routines, among others, In order to facilitate interaction within and between groups in these organizations, aspects that must be adequately aligned with organizational mission, vision and values.
\end{abstract}

Key words: management; culture management; organizations; professional sports 


\section{Introducción}

La gestión del deporte como actividad económica con fines sociales, ha sido influenciada por los conocimientos científicos y tecnológicos que suceden en un campo particular del saber, como lo es el de las Ciencias Administrativas y Gerenciales, desde donde se generan prácticas, métodos, herramientas y procedimientos, que en mayor o menor medida impactan la actual forma como se organizan y articulan las organizaciones dedicadas a la promoción, organización y administración del deporte, trayendo consecuencias en el cómo pensamos y actuamos, frente a los desafíos que trae consigo su conducción para el logro de sus objetivos. Desde luego, para que el deporte pueda direccionarse de manera exitosa "requiere de sólidas estructuras, adecuadamente gerenciadas para articular un conjunto de aspectos, donde están presentes los recursos requeridos para su práctica como escenarios deportivos, implementos, personal capacitado, apoyo financiero, entre otros" (Aguilar, 2014, p. 51).

Aun así, las organizaciones del deporte tienen culturas diferentes que se configuran en estructuras y sistemas; culturas que tienen su influencia con el pasado en cuanto a normas, rutinas, hábitos, entre otros, y también con el presente, producto de las nuevas tecnologías vinculadas al trabajo, a los objetivos organizacionales y al perfil de las personas que operan en ellas (Gómez, 2017).

A este respecto, Radcliffe-Brown (1975) citado por Abarbanel (2002), señala que la cultura es la adquisición de características mentales y hábitos que capacitan a los individuos para participar en un sistema social que les permite mantener el orden y generar mecanismos de adaptación para conservar el equilibrio entre la comunidad y su ambiente.

Por su parte, Boas (2008), sostiene que la cultura son rasgos conductuales que se aplican de forma coherente e históricamente particulares, y que esa cultura solamente puede ser entendida desde adentro, vale decir en sus propios términos, en donde la socialización y el uso de un lenguaje distintivo tienen cardinal importancia.

Es evidente entonces, que contar con una sólida cultura en las organizaciones que promocionan y desarrollan el deporte es vital si realmente se aspira ser eficaz, eficiente y transparente en la gestión, en el caso que nos ocupa, la cultura gerencial con respecto a la conducción efectiva de las organizaciones del deporte, siendo conveniente precisar el término gerencia. Kotter (2007) sostiene que la gerencia está orientada a manejar la complejidad de los procesos administrativos, garantizando sistemáticamente la congruencia entre los planes y sus resultados, y la rigidez de sus estructuras organizativas. Esta definición se complementa con aquella referida específicamente a la gerencia del deporte, que se expresa como:

... el proceso de planificar y llevar a la práctica la amplitud de acciones, variedad de competencias y ejercicios de la totalidad de los estamentos y de los miembros de la organización deportiva, de forma coordinada y racional, buscando (...) la eficacia colectiva, la cualidad de los resultados y la rentabilidad de los recursos invertidos (Mestre, 2006, p. 9)

En consecuencia, es imperativo que un gerente combine las características propias del rol relacionadas con la autoridad y el poder formal que se le otorga para planificar, estructurar y 
sistematizar las complejidades de una organización, con aquellos rasgos diferenciadores que envuelven su cultura, donde entran en juego aspectos de características subjetivas como las emociones, las motivaciones, los hábitos, las rutinas, las costumbres, en sí, los modos de sentir y pensar que determinan las acciones, facilitando "las condiciones para que los recursos humanos de la organización respondan individual y creativamente a un medio que requiere adaptaciones permanentes" (Klisberg, 2000, citado por Rusque, 2008, p. 11); aspectos que en definitiva contribuyen a reorientar comportamientos conducentes al logro de los objetivos y metas organizacionales.

Abundando al respecto, Etking y Schvarstein (1992) entienden la cultura gerencial como los modos de pensar, creer y hacer cosas en una organización. Estos modos sociales de acción están establecidos y son aplicados por las personas mientras pertenecen a los grupos de trabajo, incluyendo formas de interacción comunicativa transmitida y mantenida en el grupo, tales como lenguajes propios del sistema, liderazgos internos o preferencias compartidas.

Para ello, es pertinente una cultura gerencial orientada a enfrentar los desafíos que impone la globalización a fin de producir aquellos cambios y transformaciones necesarias que garanticen el éxito en las organizaciones (Granell, 1997)

Así pues, estos elementos propios que están presentes en cualquier organización, se desarrollan en un marco de intercambios recíprocos con el medio social, estando asociados al comportamiento de sus miembros y forman parte de la manera de conducirse en su lugar de trabajo, postura congruente con las prescripciones de Malinowsky (1970) quien afirma que la expresión social de las necesidades de sus integrantes es el reflejo de una organización.

Sobre la base de estas consideraciones, el presente estudio tiene como objetivo conocer la visión que tienen los dirigentes del deporte de la cultura gerencial con respecto a la conducción efectiva de las organizaciones del deporte: El caso Venezuela, en atención a las cinco ligas profesionales que hoy en día funcionan, como son las ligas de baloncesto, beisbol, futbol, polo acuático y voleibol. En este sentido, el propósito es explorar las ideas visionarias de los actores que ejercen funciones gerenciales, a partir de tres perspectivas o categorías de análisis como son: (a) El Perfil Gerencial; (b) Las Características de la Cultura Organizacional; y (c) La Concepción de la Cultura Gerencial.

\section{Método}

Anteriormente, los aportes teóricos en investigaciones vinculadas a las ciencias administrativas y gerenciales habían sido formulados con un marcado predominio hacia modelos conductistas y cuantitativos, admitiendo el reduccionismo teórico y metodológico para resolver la diversidad de problemas en el campo del saber. Hoy en día, el pensamiento gerencial como fenómeno social que está emergiendo está enmarcado en los nuevos paradigmas organizativos (Corredor, 2010), donde la investigación cualitativa ha tenido una destacada tendencia, dada su naturaleza para capturar datos "blandos", vale decir, el estudio de dimensiones subjetivas como las interacciones sociales, las actitudes, los valores, el lenguaje, las creencias, los símbolos, los hábitos. 
A tal efecto, en esta investigación se dispuso conocer la visión que tienen los dirigentes del deporte de la cultura gerencial con respecto a la conducción efectiva de las organizaciones del deporte: El caso Venezuela desde una postura cualitativa, a fin de capturar aquellos datos que surgieron de la percepción que tienen los actores sociales que ejercen funciones gerenciales acerca del tema objeto de estudio, desde su consciencia, de su manera de pensar. La característica más notable de las investigaciones cualitativas es que "los datos cualitativos son, en general, todos aquellos que no se pueden expresar con números: existen en forma de palabras y pueden consistir en descripciones detalladas de situaciones, eventos, personas, interacciones y conductas (...) actitudes, creencias y pensamientos" (Patton, 1980, p. 22)

Correspondientemente, para obtener el conocimiento a partir de los datos que surgieron, el estudio estuvo apoyado en un nivel de investigación descriptiva y un diseño de investigación de campo. La investigación descriptiva, a fin de caracterizar los hechos encontrados (Arias, 2016), determinando la relación que existe entre los conceptos y categorías que fueron emergiendo de los actores sociales. El diseño de investigación de campo con el propósito de interpretar los datos obtenidos, traduciendo operativa y metodológicamente el conjunto de ideas, conceptos y representaciones que se generaron sobre el fenómeno objeto de estudio. Asimismo, esta interpretación cualitativa de los datos se trianguló estratégicamente con un análisis cuantitativo (Martínez, 2009)

Para la sistematización de los datos se utilizó la teoría fundamentada de Corbin y Strauss (2002), que permitió el ordenamiento conceptual de los datos que fueron emergiendo de la realidad estudiada. En consecuencia, el estudio se soportó en dos factores:

El método comparativo constante: Consistió en el análisis de los datos en codificación abierta para desarrollar conceptos, que luego con la comparación recurrente de estos datos, fueron refinados y clasificados en categorías. Después en codificación axial, se determinó el tipo de relación entre los conceptos y las categorías.

El muestreo teórico: Se fundamentó en la selección de nuevos informantes para ser entrevistados, donde se ampliaron aquellos conceptos que fueron desarrollados, hasta que hubo saturación teórica, es decir, no emergieron nuevos datos para ser analizados.

En este tenor, se focalizaron y abordaron doce informantes clave, con potencial y relevancia para ser entrevistados en profundidad; todos ellos con conocimientos, status, destrezas comunicativas especiales, además de poseer o haber tenido cargos directivos en las ligas deportivas profesionales en Venezuela, actores que estuvieron siempre dispuestos a cooperar con el investigador (Tabla 1)

Tabla No. 1. Organizaciones objeto de estudio e informantes clave 


\begin{tabular}{|c|c|c|}
\hline ORGANIZACIÓN & INFORMANTE & CARGO \\
\hline \multirow{2}{*}{$\begin{array}{l}\text { LIGA PROFESIONAL DE } \\
\text { BALONCESTO }\end{array}$} & Rostin González & Gerente General de Cocodrilos de Caracas \\
\hline & Argenis Mujica & Gerente Deportivo de Panteras de Miranda \\
\hline \multirow{3}{*}{$\begin{array}{l}\text { LIGA VENEZOLANADE } \\
\text { BEISBOL PROFESIONAL }\end{array}$} & Oscar Prieto & Presidente de la Liga de Beisbol Profesional \\
\hline & Ariany Pagadizabal & Gerente de Mercadeo de Los Leones del Caracas \\
\hline & Samuel Moscatel & Gerente Deportivo de Caribes de Oriente \\
\hline \multirow{3}{*}{$\begin{array}{l}\text { LIGA VENEZOLANADE } \\
\text { FÚTBOL }\end{array}$} & Alejandro Batista & Gerente de Comercialización Caracas Futbol Club \\
\hline & Ignacio Sanglade & Ex Presidente Comisión de Finanzas FVF \\
\hline & Karim Assafo & Director General Deportivo Táchira Futsal \\
\hline \multirow{2}{*}{$\begin{array}{l}\text { LIGA NACIONAL DE } \\
\text { POLO ACUÁTICO }\end{array}$} & Carlos Jiménez & Ex Presidente de la Liga de Polo Acuático \\
\hline & Joaquín López & Vice Presidente de Delfines de Aragua \\
\hline \multirow{2}{*}{$\begin{array}{c}\text { LIGA VENEZOLANA DE } \\
\text { VOLEIBOL }\end{array}$} & Juan Carlos Blanco & Presidente de la Liga de Voleibol \\
\hline & Oswaldo Borges & Asesor de la Liga de Voleibol \\
\hline
\end{tabular}

\section{Resultados y Discusión}

\section{Análisis bajo codificación abierta y codificación axial}

Para el procesamiento y análisis de los datos que permitieron conocer la visión que tienen los dirigentes del deporte de la cultura gerencial con respecto a la conducción efectiva de las organizaciones del deporte: El caso Venezuela, fue necesario realizar el ordenamiento conceptual en unidades de análisis, representadas por las cinco ligas deportivas profesionales. Como se muestra en la tabla 2, en estas unidades de análisis emergieron en el proceso de codificación abierta un total de 82 etiquetas conceptuales, las cuales fueron clasificadas en tres categorías de acuerdo a sus propiedades, como son:

$\checkmark$ Perfil Gerencial: Rasgos asociados al ejercicio gerencial que aseguran comportamientos deseables para un desempeño exitoso.

$\checkmark$ Características de la Cultura Organizacional: Atributos de un sistema determinado por el conjunto de relaciones interpersonales existentes en la organización en conexión con la filosofía organizacional.

$\checkmark$ Concepción de la Cultura Gerencial: Postura que se tiene respecto a la manera de ver y concebir el quehacer gerencial desde la perspectiva de una cultura orientada a generar cambios personales, grupales y organizacionales.

Tabla No. 2. Ordenamiento conceptual en codificación abierta

\begin{tabular}{|c|c|c|c|c|}
\hline \multirow{2}{*}{$\begin{array}{l}\text { Unidades de } \\
\text { Análisis }\end{array}$} & \multicolumn{3}{|c|}{ Categorías } & \multirow[t]{2}{*}{ TOTAL } \\
\hline & $\begin{array}{c}\text { Perfil } \\
\text { Genencial }\end{array}$ & $\begin{array}{c}\text { Características de la cultura } \\
\text { Organizacional }\end{array}$ & $\begin{array}{l}\text { Concepción de la } \\
\text { cultural general }\end{array}$ & \\
\hline $\begin{array}{l}\text { Liga Profesional de } \\
\text { Baloncesto }\end{array}$ & 3 & 4 & 4 & \\
\hline $\begin{array}{c}\text { Liga Venezolana de } \\
\text { Beisbol }\end{array}$ & 7 & 6 & 9 & \\
\hline $\begin{array}{c}\text { Liga Venezolana de } \\
\text { Futbol }\end{array}$ & 6 & 3 & 11 & \\
\hline $\begin{array}{l}\text { Liga Nacional de } \\
\text { Polo Acuático }\end{array}$ & 3 & 3 & 10 & \\
\hline $\begin{array}{c}\text { Liga Venezolana de } \\
\text { Voleibol }\end{array}$ & 5 & 2 & 6 & \\
\hline Total & 24 & 18 & 40 & 82 \\
\hline
\end{tabular}




\begin{tabular}{lllll}
\hline$\%$ & $29.27 \%$ & $21.95 \%$ & $48.78 \%$ & $100 \%$ \\
\hline
\end{tabular}

Estos datos revelan que la categoría "Concepción de la cultura gerencial” aglutinó la mayor cantidad de etiquetas conceptuales con 40, que representaron el 48,78\% del total de etiquetas que emergieron durante el proceso de análisis bajo esta modalidad de codificación. Asimismo, fue la categoría que obtuvo mayor cantidad de etiquetas conceptuales en cada unidad de análisis.

Estos datos dan cuenta de la importancia que otorgan los actores sociales a concebir una cultura gerencial que contribuya a la integración de todos los miembros de los equipos que pertenecen a las ligas, la cual debe permear desde la alta gerencia hasta los jugadores, una cultura gerencial que influya inclusive en aquellos directivos de incursión reciente en las ligas deportivas profesionales y que estén alineados con la filosofía organizacional, vale decir, su misión, visión y valores, asumiendo el compromiso para garantizar el éxito deportivo.

Seguidamente se consolidaron los datos de todas las unidades de análisis en codificación axial para determinar el tipo de relación sobre la base de los siguientes criterios:

$\checkmark$ Es una Propiedad: Rasgos distintivos que identifican y se diferencian del resto de los tipos de relación, caracterizando la esencia de un concepto dentro de una categoría.

$\checkmark$ Es una Consecuencia: Aquel concepto que se produce como resultado de una acción o hecho derivado de una categoría.

$\checkmark$ Es parte: Aun cuando no es un rasgo distintivo, es un componente o segmento de un concepto con respecto a la categoría.

En este tenor, en la tabla 3 se puede apreciar el consolidado de los datos surgidos en codificación abierta, que al relacionarlos en codificación axial, permitieron determinar el tipo de relación que tuvieron las 82 etiquetas conceptuales con respecto a las tres categorías seleccionadas.

Tabla No. 3. Consolidado de tipos de relación de etiquetas conceptuales con categorías en codificación axial

\begin{tabular}{|c|c|c|c|c|}
\hline \multirow[t]{2}{*}{ Categorías } & \multicolumn{3}{|c|}{ Tipos de Relación Etiquetas } & \multirow[t]{2}{*}{ TOTAL } \\
\hline & Propiedad & Consecuencia & PARTE & \\
\hline Perfil Gerencial & 18 & 1 & 5 & \\
\hline Características de la cultura Organizacional & 7 & 6 & 5 & \\
\hline Concepción de la cultural general & 15 & 17 & 8 & \\
\hline Total & 40 & 24 & 18 & 82 \\
\hline$\%$ & $42.78 \%$ & $29,26 \%$ & $21,96 \%$ & $100 \%$ \\
\hline
\end{tabular}

De esta tabla se desprenden cifras de interés, donde se ha estimado segmentarlas para una mejor comprensión. En tal sentido, la figura 1 muestra las tendencias en los tipos de relación de las etiquetas conceptuales con las categorías. En primera instancia, se puede observar que en el tipo de relación de las etiquetas conceptuales con cada una de las categorías, la categoría "Perfil Gerencial" obtuvo la mayor cantidad de etiquetas conceptuales en el tipo de relación "Es una propiedad" con 18, seguida de la categoría "Concepción de la Cultura Gerencial" en los tipos de relación "Es una consecuencia" y "Es una propiedad" con 17 y 15 etiquetas conceptuales respectivamente. 


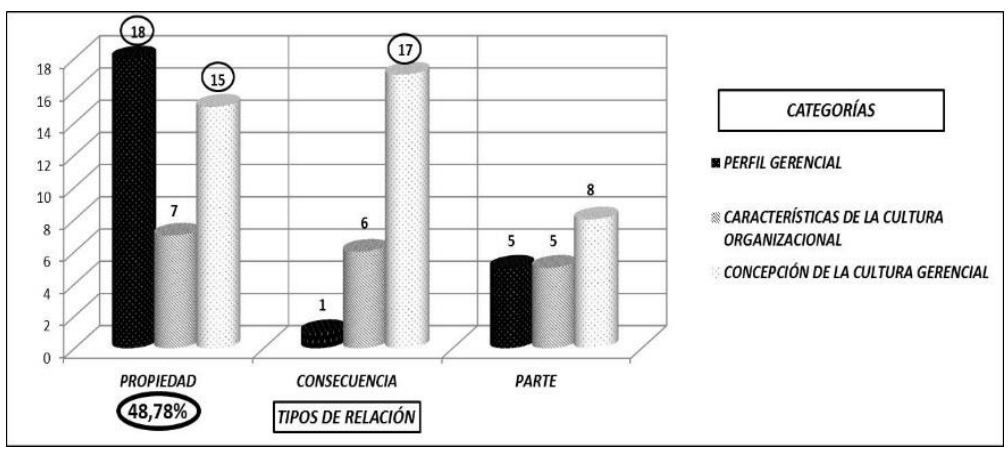

Figura No. 1. Tendencias en los tipos de relación de las etiquetas conceptuales con las categorías

Estos datos apuntan a percibir un Perfil Gerencial y una Concepción de la Cultura Gerencial en una relación de propiedad, dado a que sus datos caracterizan en su esencia a estas dos categorías. Por una parte, un perfil gerencial que debiera poseer formación universitaria, experiencia acumulada dentro del sector deportivo, sólidos valores que permitan unir esfuerzos para la consecución de objetivos y metas organizacionales, además de las competencias gerenciales necesarias para el ejercicio de sus funciones; y por otra parte, una Concepción de la Cultura Gerencial que se identifique con la filosofía organizacional, que se conduzca con responsabilidad, tolerancia y respeto, y que las acciones gerenciales estén condicionadas por unas funciones bien definidas, sin que ello implique no ser inflexible.

Asimismo, los datos revelan que las etiquetas conceptuales relacionadas con la categoría Concepción de Cultura Gerencial, también debieran ser producto de las consecuencias que se derivan de las acciones de un gerente, las cuales se sintetizan en la influencia que pudiesen ejercer los directivos al resto de la organización, dentro de un clima que garantice relaciones horizontales y flexibles, donde se perciba un liderazgo de calidad, materializado en transmitir confianza y que a su vez eleven el compromiso de las personas.

Además de lo anterior, el mismo gráfico también exhibe la tendencia del tipo de relación prevaleciente en el total de relaciones entre etiquetas conceptuales y categorías. En efecto, se puede evidenciar que las cifras se inclinan hacia el tipo de relación "Es una propiedad", la cual obtuvo $48,78 \%$ del total de enlaces. Datos que permiten inferir el peso que se confiere a aspectos propios de los conceptos emergentes en las tres categorías seleccionadas en este estudio.

Así pues, se aspira como visión un Perfil Gerencial, unas Características de la Cultura Organizacional y una Concepción de la Cultura Organizacional que en su esencia sirvan de plataforma y mecanismo influenciador para orientar una cultura gerencial que se conduzca con efectividad en las organizaciones del deporte profesional en Venezuela, con énfasis en las ligas deportivas profesionales.

\section{Interpretación de los hallazgos encontrados}

A la luz del análisis desarrollado en codificación abierta y codificación axial, se procedió a interpretar los hallazgos encontrados en cada una de las cinco ligas deportivas profesionales que se generaron a través de la interacción con los actores sociales que ejercen funciones gerenciales, con la intención de conocer la visión que tienen los dirigentes del deporte de la 
cultura gerencial con respecto a la conducción efectiva de las organizaciones del deporte: El caso Venezuela. En este sentido, los hallazgos permitieron derivar las siguientes interpretaciones:

\section{Liga Profesional de Baloncesto:}

En relación con el Perfil Gerencial, se detectó que para ejercer funciones gerenciales, su perfil debe estar condicionado por un actor que provenga del deporte, con la necesaria experiencia desde el rol de atleta, entrenador o dirigente dentro de la organización; experiencia que debiera traducirse en años acumulados y que a su vez pueda conjugarse con la formación académica universitaria.

En cuanto a las Características de la Cultura Organizacional, se desea una organización cuya gestión esté centrada en el deporte y no exclusivamente en lo económico, con una visión, un sistema de valores y unas creencias que sean compartidas por todos sus miembros, garantizando en las bases deportivas el estudio y la formación académica, muy bien compaginadas con la práctica deportiva.

Con respecto a la Concepción de la Cultura Gerencial, la postura que prevalece es que ella debe velar por la integración de todos los miembros que hacen vida dentro de la organización, donde la alta gerencia proyecte una imagen deseable hacia las personas, mediante el modelaje de valores organizacionales y a través de sus acciones.

\section{Liga venezolana de Béisbol Profesional:}

En referencia con el Perfil Gerencial, existe una marcada exigencia por disponer de un actor que simbolice un comportamiento con suficiente liderazgo, arraigo y sentido de pertenencia hacia la organización, comportamiento que debiera estar consustanciado con sólidos valores que sirvan de guía a todos los involucrados para capitalizar en acciones efectivas mediante el esfuerzo en conjunto. Asimismo, el candidato deseable tendría que poseer experiencia en el ámbito deportivo, formación académica y conocimientos gerenciales para garantizar eficiencia y eficacia en la gestión; en definitiva, competencias gerenciales necesarias para poder operar con efectividad en las funciones, responsabilidades y tareas inherentes al desempeño gerencial.

En correspondencia con las Características de la Cultura Organizacional, se aspira principalmente una organización moderna y ajustada a estándares mundiales, cuyas cualidades estén determinadas por una gestión altamente profesional, orientando de manera recurrente y sostenida los procesos de capacitación y funcionando en sinergia cooperativa con todos los equipos profesionales.

En lo atinente a la Concepción de la Cultura Gerencial, se resalta la aspiración de un comportamiento enfocado en un liderazgo que promueva el fanatismo y la pasión de sus miembros hacia la organización, garantizando que tanto la alta gerencia, peloteros y trabajadores en general estén comprometidos para alcanzar el éxito deportivo. Ello implicaría una cultura gerencial que esté orientada por la misión, visión y valores, a los fines de dar cumplimiento con los objetivos y metas organizativas, lo que supondría cultivar relaciones armoniosas, horizontales y flexibles entre directivos y jugadores, sin que se dejen de reconocer las deficiencias o limitaciones que puedan presentarse, asegurando el necesario acompañamiento gerencial, 
aspectos que en definitiva permitirían elevar un quehacer gerencial que se aproxime a un modelo de equipo como los que operan en las Grandes Ligas del Béisbol de los Estados Unidos.

\section{Liga Venezolana de Futbol:}

En concordancia con el Perfil Gerencial, se manifiesta el deseo por una práctica gerencial que promueva la búsqueda permanente de líderes identificados plenamente con la organización, a los fines de garantizar la integración de las personas y el trabajo en equipo. En sí, un perfil con formación académica alineada con el desempeño deseado y la necesaria experiencia para asumir nuevos retos.

Con relación a las Características de la Cultura Organizacional, se enfatiza la importancia de una gestión basada en una visión compartida y que a su vez esté soportada en un sistema de valores y creencias comunes para todos sus miembros. Asimismo, se aspira una organización que desde el deporte pueda contribuir con los cambios sociales cónsonos con las demandas de la sociedad, así como un adecuado manejo de relaciones con las otras organizaciones pertenecientes a la Liga, además de instituciones estadales, nacionales, empresariales y sociedad en general.

En cuanto a la Concepción de la Cultura Gerencial, se reitera la valoración que se tiene hacia la filosofía organizacional como hilo conductor del comportamiento gerencial, el cual debe estar orientado a generar confianza en las personas, a conducirse con responsabilidad en sus funciones y acciones, y a otorgar recompensas justas, pero también a corregir errores, desviaciones u omisiones en los procesos. En fin, una cultura gerencial caracterizada por reflejar un liderazgo que esté soportado en el respeto hacia los otros y en el modelaje permanentemente de los valores organizacionales.

\section{Liga Nacional de Polo Acuático:}

Con respecto al Perfil Gerencial, éste se traduce en la formación académica, la capacitación permanente y la experiencia en el deporte como pilares fundamentales para operar dentro del proceso gerencial con eficiencia en el manejo de los recursos y eficacia en los resultados. Con relación a las Características de la Cultura Organizacional, ésta debiera consolidar un desarrollo organizacional que apueste a una visión compartida y con unos valores consustanciados con la práctica deportiva, valores que impliquen al mismo tiempo el estímulo hacia la educación y la formación como factores a considerar en la cultura organizacional.

En conformidad con la Concepción de la Cultura Gerencial, se tiene la percepción que la cultura debe estar determinada por un conjunto de reglas y normas organizativas que se deben respetar, mediante una estructura funcional que dé dirección y sentido a las acciones gerenciales en la búsqueda de lograr la integración del conjunto de personas dentro de la organización. En sí, una cultura gerencial articulada con la misión, visión, valores y objetivos estratégicos claros, impulsada desde la gerencia a los fines de garantizar el éxito organizacional.

\section{Liga Venezolana de Voleibol:}

Con relación al Perfil Gerencial, la tendencia en este sector se centró básicamente en un actor que esté dispuesto a conducirse a través de valores comprobados para el ejercicio gerencial. Ello implicaría un desempeño que sirva de guía para el resto de la organización, a los efectos de 
favorecer la confianza mediante el respeto hacia las personas, siendo honesto y equitativo en sus acciones y modelando un comportamiento que facilite la integración de la gente para garantizar el logro de los objetivos y metas organizativas.

En correspondencia con las Características de la Cultura Organizacional, éstas se focalizaron en la aspiración de consolidar una organización que pueda desarrollarse y crecer a través de una filosofía de gestión, la cual debe estar orientada a favorecer el clima organizacional a través de una visión que cuente con el respaldo de su gente, que al ser compartida inspire a todos con el interés de lograr resultados cónsonos con lo esperado. Asimismo, es fundamental que en las bases deportivas se valore y articule la formación académica con el deporte.

En lo atinente a la Concepción de la Cultura Gerencial, se considera que ésta al impactar en gran medida en las acciones, conviene colocar el foco en el seguimiento, monitoreo y control de aquello que ha sido planificado para evitar desviaciones que pudieran comprometer su cumplimiento y así responder con la debida efectividad. A tal efecto, es imperativa una cultura gerencial que pueda dar direccionalidad a una filosofía organizacional que envuelve a la misión, visión y valores, a través del esfuerzo conjunto de las personas para garantizar el éxito organizativo.

\section{Conclusiones y Recomendaciones}

A la luz de las evidencias y hallazgos encontrados en este estudio, en torno a la visión que tienen los dirigentes del deporte de la cultura gerencial con respecto a la conducción efectiva de las organizaciones del deporte: El caso Venezuela, se pudo constatar que los gerentes pertenecientes a las cinco ligas deportivas profesionales esperan una concepción filosófica que esté determinada por un conjunto de percepciones, emociones, actitudes, hábitos, creencias, valores, tradiciones, rutinas y formas de interacción dentro y entre los grupos existentes en estas organizaciones.

Todo este conjunto de aspectos, adecuadamente alineados con la filosofía organizacional -determinada por la misión, visión y valores-, darían dirección y sentido a las acciones, garantizando el cumplimiento de objetivos y metas organizacionales mediante el acompañamiento gerencial centrado en el respeto, la honestidad, la equidad, la integración y el sentido de pertenencia, y con competencias gerenciales adquiridas a través de los años en las organizaciones del deporte.

Asimismo, los actores entrevistados demandan un comportamiento gerencial que garantice el éxito de sus respectivos equipos. A tal efecto, se considera pertinente por una parte la formación académica en las personas que realizan funciones gerenciales, y por otra, que el gerente de estas organizaciones tenga la capacidad de adaptación a los cambios y transformaciones que reclama el deporte profesional. En este sentido, la mirada que tienen los gerentes de la cultura gerencial con respecto a la conducción efectiva de las organizaciones del deporte: El caso Venezuela, de algún modo muestra la manera de ver y comprender una realidad deseada por estos actores, un "deber ser" que es necesario contrastar con los patrones de comportamiento que prevalecen en el ejercicio gerencial de estas organizaciones, a los fines de determinar las brechas que existen entre una cultura gerencial que se aspira tener -como se evidenció en este estudio- y lo que verdaderamente ocurre en la realidad. 


\section{Bibliografía}

Abarbanel, A. (2002). Cultura Organizacional: Aspectos teóricos prácticos y metodológicos. España: Legis.

Aguilar, P. (2014). Gerencia del deporte de alto rendimiento en Venezuela: hacia una implantación efectiva de los procesos administrativos. Saarbtücken: Editorial Publicia.

Arias, F. (2016). El Problema de Investigación: Introducción a la Metodología Científica. Caracas: Editorial Episteme.

Boas, F. (2008). Textos de antropología. Madrid: Editorial Centro de Estudios Ramón Areces.

Corbin, J. y Strauss, A. (2002). Bases de la investigación cualitativa: técnicas y procedimientos para desarrollar la teoría fundamentada. Medellín: Universidad de Antioquia.

Corredor, J. (2008). Relaciones públicas y los nuevos paradigmas organizativos. Cuadernos de Postgrado: Gerencia y Condición Humana. 28, 121-131.

Gómez, S. (2017). Cultura gerencial en las organizaciones del deporte: Valores, creencias, prácticas, herramientas, métodos y procedimientos en organizaciones del deporte. Saarbrücken: Editorial Académica Española.

Granell, E. (1997). Éxito Gerencial y Cultura: Retos y oportunidades para Venezuela. Caracas: Instituto de Estudios Superiores de Administración-IESA.

Etking, J. y Schvarstein, L. (1992). La Identidad de las Organizaciones. Buenos Aires: Paidós.

Klisberg, B. (2000). Una nueva gerencia pública para la modernización del Estado y enfrentar los desafios de la globalización. Disponible en: http://orion2020.org/archivo/competencias_gerenciales/comunicacion_organizacional/s2_nue vagerenciapublica.pdf

Kotter, J. (2007). Al frente del cambio. México: Editorial Empresa Activa.

Malinoswski, B. (1970). Una teoría científica de la cultura y otros ensayos. Buenos Aires: Sudamericana.

Martínez, M. (2009). Ciencia y arte en la metodología cualitativa. México: Trillas.

Mestre, J. (2006). El Procés de Panificació en la Gestió de L"esport Municipal. En: F. Calabuig (Coord.) Propostes de Planificació i organización de I"e esport Municipal (pp. 7-52). Valencia.

Patton, M (1980). Métodos de evaluación cualitativa. Beverly Hills: Sage.

Radcliffe-Brown, A.R. (1975). El método en Antropología social. Barcelona: Anagrama.

Rusque, A. (2008). Empresarios y gerentes: sujetos en formación en la educación superior. Cuadernos de Postgrado: Gerencia y Condición H 